\title{
Reviewers 2012
}

We would like to thank the following reviewers who have taken part in the peer-reviewing process for ADHD Attention Deficit and Hyperactivity Disorders in 2012. Their efforts and advice essentially contributed to maintain the high quality of the journal.

\author{
Anastopoulous, Arthur \\ Antshel, Kevin M. \\ Bau, Claiton \\ Bellgrove, Mark \\ Blanz, Bernhard \\ Buchhorn, Rainer \\ Bundy, Anita \\ Catania, Charles \\ Coghill, David \\ Corominas Roso, Margarida \\ Davids, Eugen \\ Dittmann, Ralf \\ Falissard, Bruno \\ Faraone, Stephen \\ Gift, Thomas \\ Gille, Gabriele \\ Gordon, Michael \\ Groen, Yvonne \\ Gundelfinger, Ronnie \\ Harrison, Allyson \\ Henessy, Karen \\ Higbee, Thomas
}

Holtmann, Martin

Hoza, Betsy

Huizinga, Mariëtte

Jacob, Christian

Jans, Thomas

Kollins, Scott

Madden, Gregory

Malone, Patrick

Manor, Iris

Marchant, Barrie

Mautone, Jennifer

Michel, Tanja

Miller, Carlin

Mitchell, John

Musso, Mandi Wilkes

Newcorn, Jeffrey

Nigg, J.T.

Philipsen, Alexandra

Polanczyk, Guilherme

Power, Thomas

Retz, Wolfgang

Rommelse, Nanda
Rosler, Michael

Russell, Vivienne

Salum, Giovanni

Shaw, Philip

Sobanski, Esther

Stein, Mark

Stoerk, Dagmar

Schwenck, Christina

Taurines, Regina

Thissen, Andrieke

Thome, Johannes

Toplak, Maggie

Vaneck, K.

von Gontard, Alexander

Wagner, Flavia

Watanabe, Shigeru

Weisler, Richard

Wilens, Timothy

Young, Susan

Zepf, Florian 